\title{
Time to adjust aspirin dose to body weight
}
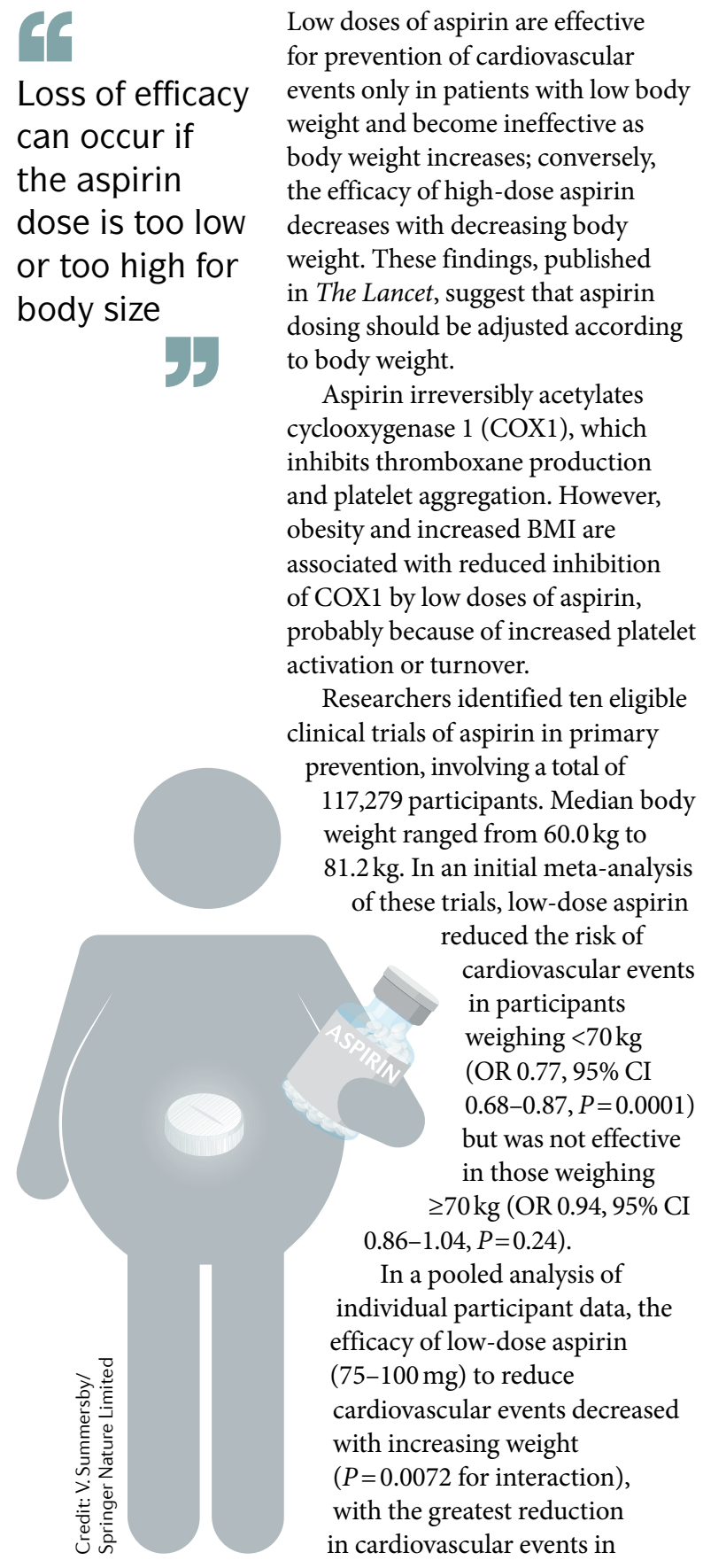

participant who weighed $50-69 \mathrm{~kg}$ (HR 0.75, 95\% CI 0.65-0.85, $P<0.001)$. Low-dose aspirin prevented stroke in women but not in men, but no significant difference remained after accounting for body weight. The effect of low-dose aspirin on cardiovascular events was not modified by the presence of diabetes mellitus or by age, but was reduced in smokers ( $P=0.0026$ for interaction). In participants weighing $\geq 70 \mathrm{~kg}$, low-dose aspirin was associated with an increase in case fatality of first cardiovascular events (OR 1.33, 95\% CI 1.08-1.64, $P=0.0082$ ).

The efficacy of higher doses of aspirin to reduce the risk of cardiovascular events increased with body weight ( $P=0.017$ for interaction). In primary prevention, $325 \mathrm{mg}$ of aspirin reduced cardiovascular events in participants weighing $\geq 70 \mathrm{~kg}$ (HR 0.83, 95\% CI 0.70-0.98, $P=0.028$ ), and $500 \mathrm{mg}$ of aspirin reduced cardiovascular events or death in those weighing $\geq 90 \mathrm{~kg}$ (HR 0.52, 95\% CI 0.30-0.89, $P=0.017)$.

Of note, low-dose aspirin reduced the risk of colorectal cancer in participants weighing $<70 \mathrm{~kg}$ (HR 0.64, 95\% CI $0.50-0.82, P=0.0004)$ but not in those weighing $\geq 70 \mathrm{~kg}$ (HR 0.87 , 95\% CI 0.71-1.07, $P=0.32$ ).

"Loss of efficacy can occur if the aspirin dose is too low or too high for body size, and other harms appear to result from excess dosing," conclude the researchers. "Reductions in cardiovascular events and all-cause death at optimal doses for weight were substantial, highlighting the potential of more tailored aspirin dosing." The investigators point out that weight-based dosing is already commonplace for thrombolytics and intravenous antiplatelet treatment, but not for oral antiplatelet drugs.

"The finding that optimal dosing of aspirin for prevention of cardiovascular events (and even cancer) depends on body weight, particularly lean body mass and height, is biologically plausible and argues for more tailored and personalized decision-making about the dose of aspirin," comments JoAnn Manson (Brigham and Women's Hospital, USA), who was not involved in the study. "Some of the sexual dimorphism observed in response to aspirin may be due to differences in body size, distribution volumes, and systemic bioavailability," continues Manson, who was a co-investigator on the Women's Health Study and US Physicians' Health Study aspirin trials.

"Despite increasing attention to the importance of precision medicine ... readily available information such as height, weight, body size, age, and sex is often overlooked. Hopefully, this report will help to usher in a new era of personalized medicine that considers readily available clinical factors, as well as molecular data, in clinical decision-making," concludes Manson.

Gregory B. Lim

ORIGINAL ARTICLE Rothwell, P. M. et al. Effects of aspirin on risks of vascular events and cancer according to bodyweight and dose: analysis of individual patient data from randomised trials. Lancet https://doi.org/10.1016/ S0140-6736(18)31133-4 (2018) FURTHER READING Capodanno, D. et al. Aspirin-free strategies in cardiovascular disease and cardioembolic stroke prevention. Nat. Rev. Cardiol. 15, 480-496 (2018) 